\title{
Overcoming non-local effects and Brillouin threshold limitations in Brillouin distributed sensors
}

\author{
Javier Urricelqui ${ }^{a}$, Rubén Ruiz-Lombera ${ }^{b}$, Mikel Sagues $^{a}$, Jesús Mirapeix $^{b}$, \\ José M. López-Higuera ${ }^{b}$ and Alayn Loayssa ${ }^{a}$ \\ ${ }^{a}$ Universidad Pública de Navarra, Pamplona, Spain; \\ ${ }^{b}$ Universidad de Cantabria, Santander, Spain
}

\begin{abstract}
We demonstrate, for the first time to our knowledge, a Brillouin optical time domain analysis sensor that is able to operate with a probe power larger than the Brillouin threshold of the deployed sensing fiber and that is free from detrimental non-local effects. The technique is based on a dual-probe-sideband setup in which a frequency modulation of the probes waves along the fiber is introduced. This makes the frequency of maximum interaction between pump and probes to vary along the fiber, thus mitigating the pump pulse depletion and making it possible to use very large probe power, which brings an improved signal-to-noise ratio in detection.
\end{abstract}

Keywords: Brillouin distributed sensors, Brillouin optical time domain analysis, Brillouin threshold

\section{INTRODUCTION}

Brillouin optical time domain analysis sensors (BOTDA) are very interesting for the measurement of temperature and strain along large lengths of singlemode optical fiber (SMF). One of the main limitations to the deployment of such long-range BOTDAs comes from so-called non-local effects, which are induced by the depletion of the pump pulses along the fiber as they transfer energy to the amplified probe wave. ${ }^{1}$ This energy transfer depends on the optical frequency of the probe and hence introduces a systematic error in the measurement of the Brillouin frequency shift (BFS). Altogether, this results in a limit to the maximum probe power that can be launched in the fiber and hence a reduction in detection signal-to-noise ratio. The most popular method to compensate this detrimental effect is the use of a dual-probe-sideband setup, in which two sidebands equally spaced from the pulse wavelength provide a complementary interaction, such that the energy that the pulse transfers to the lower optical frequency probe is compensated by that transferred from the upper optical frequency probe to the pulse. ${ }^{1}$ However, it has been recently shown that the probe power limit in dual-probe-sideband setup is rather limited, because of the distortion of the pump pulse spectra that is brought by the combined gain and loss spectra generated by the two probe waves. ${ }^{2}$ Therefore, the limitation to long-range BOTDAs coming from non-local effects is yet to be solved. Furthermore, even though non-local effects were completely overcome, another limit for the probe power would remain: the Brillouin threshold of the fiber. This threshold defines the maximum power that can be launched into the sensing fiber before the amplification of thermally-induced spontaneous Brillouin scattering (SpBS) leads to depletion of the probe wave and addition of noise to the detected signal. ${ }^{3}$

In this work, we introduce a method to greatly expand the probe power that can be deployed in BOTDA sensors, overcoming previous non-local effect limits and even the Brillouin threshold limit. It is based on modulating the optical frequency of the probes in a dual-probe setup.

\section{DESCRIPTION OF THE TECHNIQUE}

Fig. 1 shows the fundamentals of the technique by depicting the spectra of the various optical waves involved. A BOTDA setup with two probe waves counter-propagating to the pump pulse is assumed. As it is highlighted in the figure, the optical frequency of the probe waves is modulated in the time domain. Moreover, this frequency modulation (FM) is synchronized to the pump pulses so that a sequence of pulses experiences the same wavelength of the probe waves at any given location. Therefore, the modulation of the optical frequency of the probes waves

E-mail: alayn.loayssa@unavarra.es

24th International Conference on Optical Fibre Sensors, edited by Hypolito José Kalinowski, José Luís Fabris, Wojtek J. Bock, Proc. of SPIE Vol. 9634, 963487 • @ 2015 SPIE CCC code: $0277-786 X / 15 / \$ 18 \cdot$ doi: $10.1117 / 12.2205448$

Proc. of SPIE Vol. $9634963487-1$ 
leads to a variation of the Brillouin interaction frequency along the fiber that follows the same profile of this modulation. This has important implications for the interaction between pump pulse and probe waves, as its schematically depicted in Fig. 1(a). The modulation of the probe wave's optical frequency makes the Brillouin interaction induced by these waves upon the optical pulse to spread over a large frequency range so that nonlocal effects cease to be significant. Indeed, the lower frequency probe induces a loss spectrum for the pulse that varies along the fiber. The integration of this interaction along the fiber gives a flat wideband total depletion spectrum that does not distort the pulse spectrum. Moreover, the upper frequency probe induces a gain spectrum that integrated along the fiber gives a flat wideband total gain spectrum that compensates the pulse depletion. Therefore, the gain and loss spectra perfectly compensate for all the frequency components of the pulse, hence non-local effects are fully avoided. A triangular modulation of the probe waves frequency is assumed in Fig. 1. However, other modulation shapes are possible to optimize the objective of having the flattest possible total gain and depletion spectra so that no distortion is introduced to the pulse.

In detection, just one of the probe waves is detected, typically the lower frequency one, while the other is filtered out. In order to measure the Brillouin spectra experienced by the retained probe wave at any given location, it is necessary to scan the frequency of the probe wave at that location. In a conventional BOTDA this is done by sweeping the frequency of the probe wave. However, in our system this can be done in a much simpler way by sequentially changing the relative delay between the pump pulses and the FM wave, as it is schematically depicted in Fig. 1(b). This changes the relative location of the optical frequency profile of the probe wave along the fiber so that at any given location all frequencies are sequentially scanned.

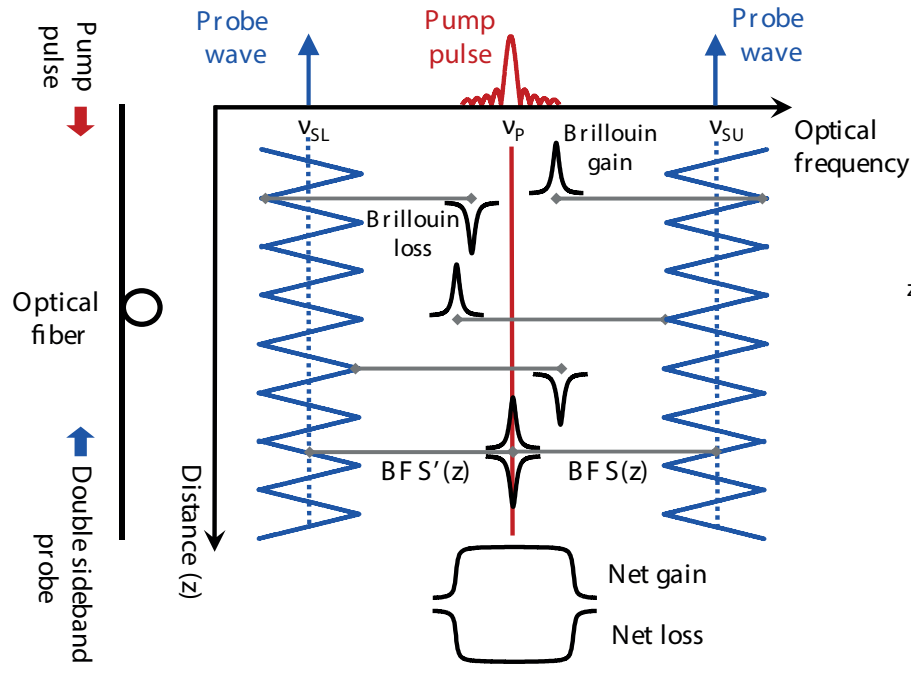

(a)

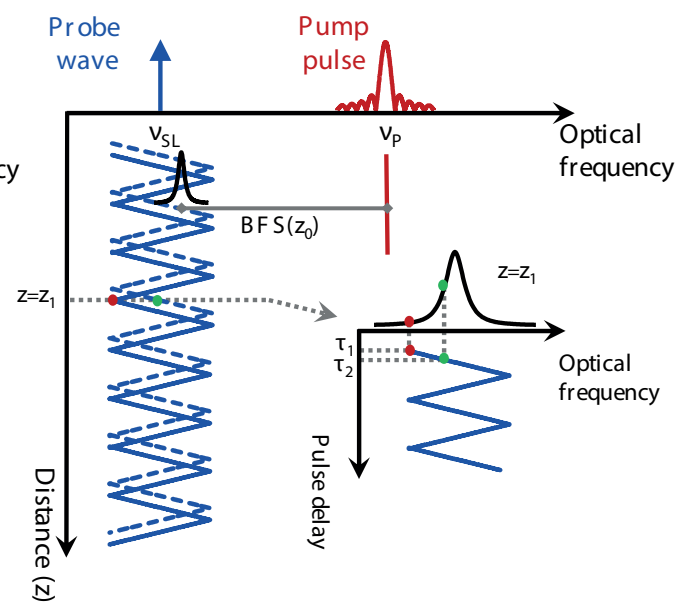

(b)

Figure 1: Fundamentals of the technique: Brillouin interaction on the pump pulse (a) and on the probe wave (b).

\section{EXPERIMENTAL RESULTS AND CONCLUSIONS}

Fig. 2 depicts the assembled setup used to demonstrate our technique. The output of a laser source is divided into two branches to generate the pump and probe waves. In the upper branch, the pump signal is pulsed using a semiconductor optical amplifier (SOA). Then, the pulse power is boosted to a peak of $140 \mathrm{~mW}$ using a erbium doped fiber amplifier (EDFA) and its polarization state is randomized using a polarization scrambler (PS) before being launched into $50 \mathrm{~km}$ of SMF. In the lower branch, a double-sideband probe wave is generated using a Mach-Zehnder electrooptic modulator (MZ-EOM) driven by an arbitrary waveform generator (AWG). The AWG provides a FM microwave signal whose instantaneous frequency varies around the average BFS of the fiber following a triangular shape and inducing an identical modulation of the optical frequency of the probe waves. After Brillouin interaction of this probe signals with the pump pulse, they are directed to a fiber Bragg 
grating (FBG) to filter out the upper sideband. The remaining probe wave is detected and captured in a digital oscilloscope.

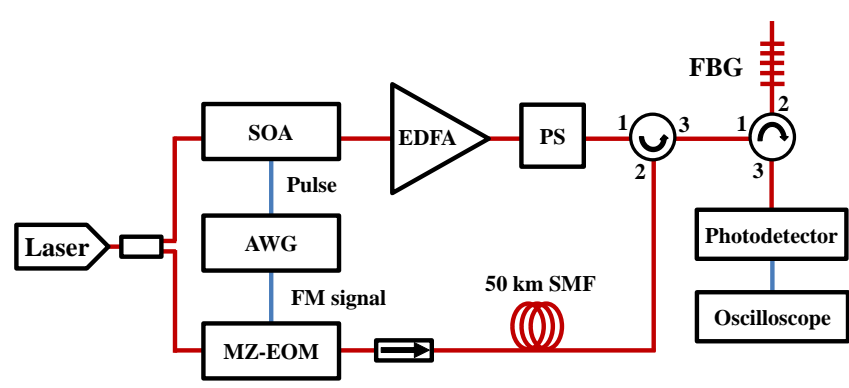

Figure 2: Experimental setup.

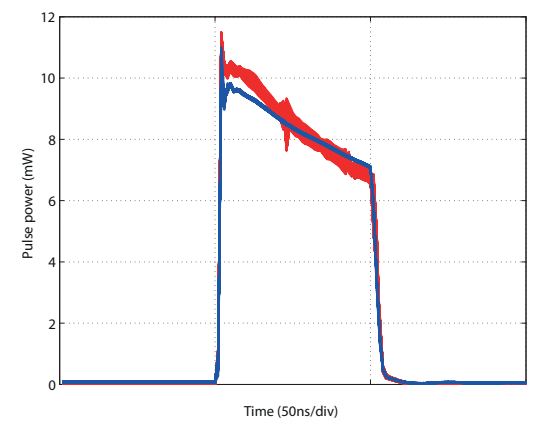

Figure 3: Pump pulses at the output of the fiber with $8-\mathrm{dBm}$ probe wave (red) or without (blue).

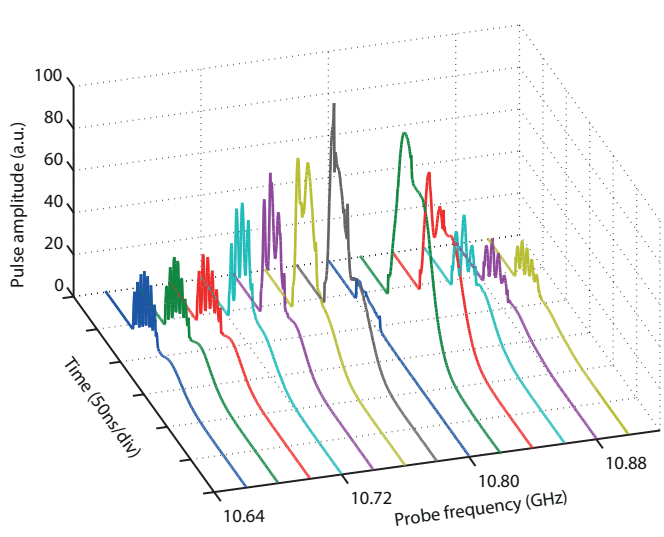

(a)

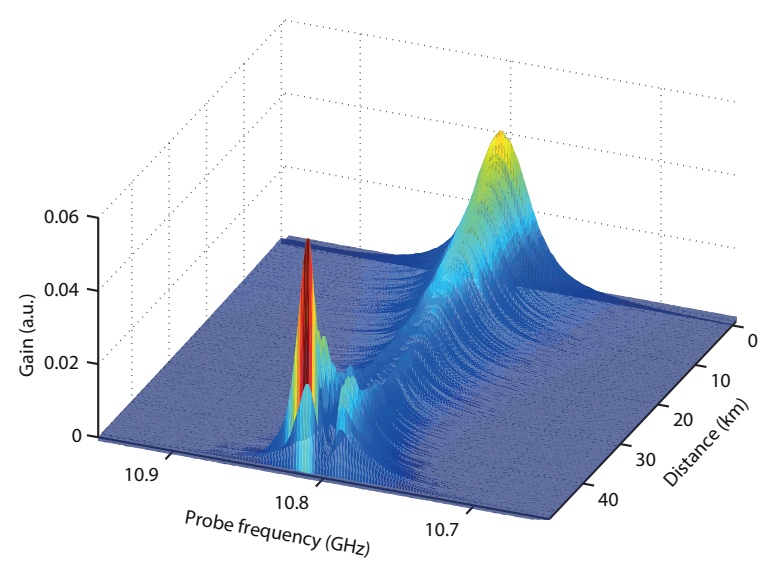

(b)

Figure 4: (a) Pulses and (b) Brillouin spectra measured for the conventional dual-probe BOTDA with 5-dBm probe power.

We started by experimentally demonstrating that our system is free from non-local effects. Fig. 3 depicts the pulses measured at the output of the fiber with an without probe wave applied. Notice that the pulse shape remains almost identical for both cases with negligible distortion and just some added noise, which is attributed to SpBS amplified by the probe waves. This measurement was performed with a probe power of $8 \mathrm{dBm}$, which, to our knowledge, is the largest probe power ever demonstrated in a long-range BOTDA sensor without errors induced by non-local effects. This performance was then compared to that obtained with a conventional dualprobe BOTDA setup. This was done by simply switching off the FM modulation on the AWG so that it generated pure microwave tones. Fig. 4(a) shows the pulses that were measured at the output of the fiber as the microwave frequency was swept to measure the Brillouin spectra. Notice that, in spite of the probe wave being $5 \mathrm{dBm}$, i.e., lower that that in the measurements of Fig. 3), the pulses are greatly distorted. This distortion has been previously reported in the literature, but for lower power of the probe wave for which the distortion effect was found to be rather mild. ${ }^{2}$ However, it can be seen that the distortion is much more pronounced as the probe wave power is increased. The gain and depletion induced by both probe waves upon the pulse compensate just when the microwave frequency coincides with the BFS of the fiber $(10.8 \mathrm{GHz})$. However, for small departures of the microwave frequency from that value, the pulse gets distorted as its spectral components get differential amplification or depletion. ${ }^{2}$ This makes the pulse peak power to greatly increase around the BFS frequency, as it can be seen in Fig. 4(a). There is also increased temporal duration of the pulses at those frequencies. Furthermore, the distortion of the pulse spectra has a strong impact on the measured Brillouin spectra along the fiber, as it can be appreciated in Fig. 4(b). This figure shows that at the far away locations of the fiber, where 


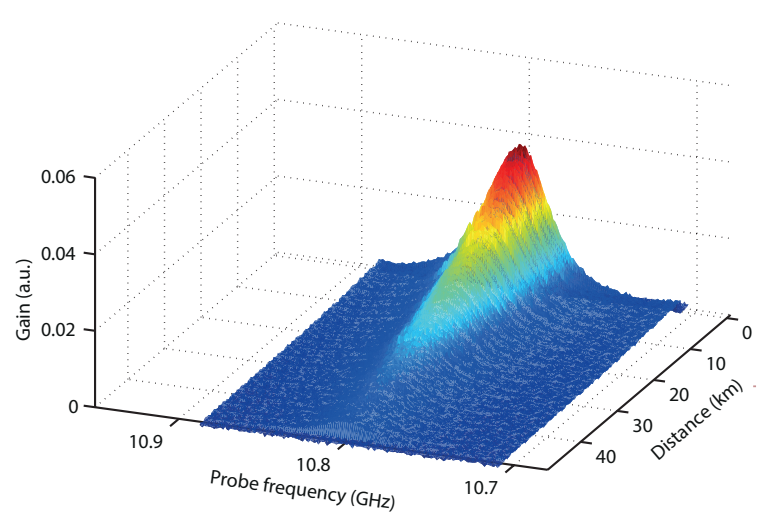

Figure 5: Brillouin spectra measured for the frequency modulation technique.

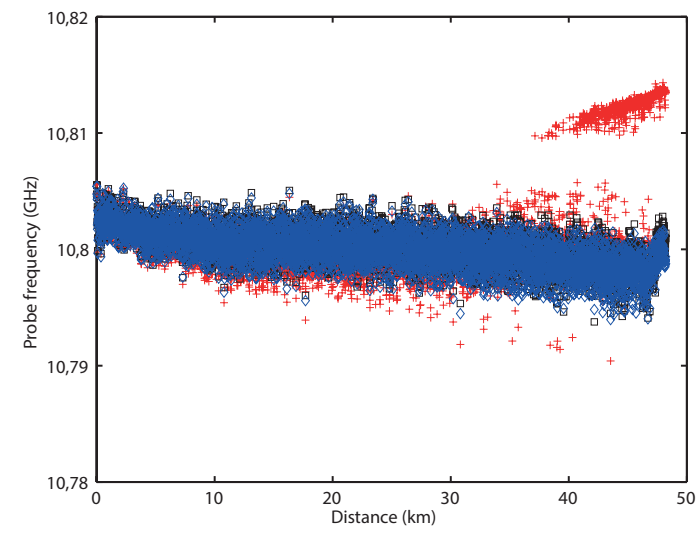

Figure 6: Comparison of measured BFS for the FM technique with 8-dBm probe power (black) and for the conventional dual probe with $5-\mathrm{dBm}$ (red) and -6-dBm (blue) probe power.

the pulse arrives with great distortion, the measured spectra is also greatly distorted. Note that in Fig. 4(b) the spectra at the end of the fiber has two peaks around the mean BFS of the fiber that correspond to the probe frequencies at which the pulse was shown to be amplified in Fig. 4(a).

In contrast to the results for the conventional dual-probe BOTDA, Fig. 5 depicts the spectra measured for our system for even larger probe wave. This spectra was obtained after post-processing of the raw measurement data to compensate the frequency shift in the measurement introduced by FM modulation of the probe wave along the fiber. It can be seen that the spectra is completely normal, not suffering any distortion. This is confirmed by Fig. 6 that displays the BFS measured for the conventional dual-probe BOTDA and for our system for different powers of the probe wave. Notice that, as it was explained before, there is a large systematic measurement error as the probe power is increased for the conventional dual-probe BOTDA, while for our system the BFS measurement remains error-free even for larger probe power.

Finally, it must be pointed out that our FM BOTDA setup was operating with a probe power above the Brillouin threshold, which was measured to be 7 -dBm for the deployed sensing fiber. This is made possible by a side effect of the FM modulation of the probe waves deployed, which has been previously shown to increase the effective Brillouin threshold. ${ }^{4}$ This was confirmed by measuring the rms noise at the detector for different probe power injected in the fiber but using an attenuator to keep a constant received power. In the conventional dual probe system there was a larger than six times increase in rms noise as the probe power was increased from $5 \mathrm{dBm}$ to $8 \mathrm{dBm}$, whereas with the FM technique no increase in noise was measured.

In conclusion, we have demonstrated a new technique for BOTDA sensors that provides an enhancement for long-range systems as larger probe power can be launched into the fiber, which leads to enhaced SNR in detection and hence improve measurement precision.

\section{REFERENCES}

[1] L. Thévenaz, S. Mafang, and J. Lin, "Effect of pulse depletion in a brillouin optical time-domain analysis system," Optics Express 21(12), pp. 14017-14035, 2013.

[2] A. Dominguez-Lopez, X. Angulo-Vinuesa, A. Lopez-Gil, S. Martin-Lopez, and M. Gonzalez-Herraez, "Nonlocal effects in dual-probe-sideband Brillouin optical time domain analysis," Optics Express 23, p. 10341, Apr. 2015.

[3] A. David and M. Horowitz, "Low-frequency transmitted intensity noise induced by stimulated Brillouin scattering in optical fibers.," Optics express 19(12), pp. 11792-11803, 2011.

[4] J. B. Coles, B. P.-P. Kuo, N. Alic, S. Moro, C.-S. Bres, J. M. C. Boggio, P. Andrekson, M. Karlsson, and S. Radic, "Bandwidth-efficient phase modulation techniques for Stimulated Brillouin Scattering suppression in fiber optic parametric amplifiers," Optics Express 18, p. 18138, Aug. 2010. 